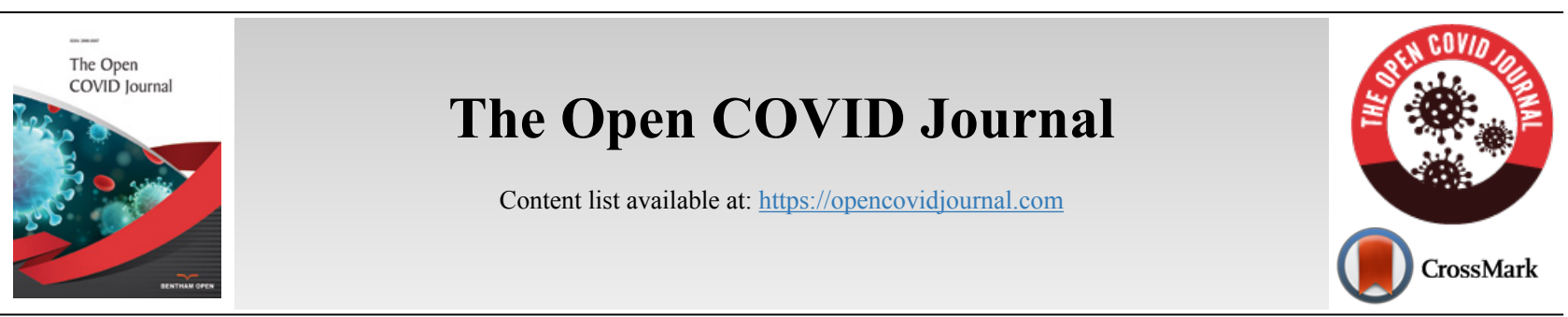

LETTER TO THE EDIT2 R

\title{
Pharmacovigilance, and COVID-19
}

\author{
Houda Filali ${ }^{1}$ and Youness Kadil ${ }^{*}, 1$ \\ ${ }^{\prime}$ Faculty of Medicine and Pharmacy of Casablanca, Laboratory of Pharmacology-Toxicology, Hassan II University of Casablanca, Casablanca \\ 20000, Morocco
}

Article History

Received: December 11, 2020

Revised: January 21, 2021

Accepted: January 27, 2021

Pharmacovigilance deals with drug-related problems: detecting, explaining and preventing adverse effects. Patient safety is the main objective of pharmacovigilance. Many approved drugs carry a risk of adverse reactions. While the benefits to the patient outweigh these risks, physicians and researchers must remain cautious of any serious side effect.

The pharmacovigilance monitoring of COVID-19 patients is an international priority. All health authorities and organizations encourage this activity in an attempt to guarantee health transparency and to be allowed to judge the benefit/risk of therapies being attempted or proposed for a specific validated treatment. Due to the spontaneous notification required for this pharmacovigilance activity, and in this particular context of the pandemic, healthcare professionals are overwhelmed by the workload. The specialized authorities are soliciting active and exhaustive data collection from the different services, receiving COVID-19 positive patients, of any suspected adverse effect that may be related to or associated with the use of any drug. This allows the detection, analysis, and evaluation of the tolerance of the drugs prescribed. In parallel, it investigates possible drug interactions resulting from drug combinations, underlying patient defects, or related to the symptomatology and/or complications of SARS-CoV-2 infection.

The surveillance of expected or unexpected effects that may appear or may be aggravated following the use of recommended drugs/combined drugs in the management of patients with COVID-19 is essential for the evaluation of tolerance and therapeutic responsiveness. It is important to mention that, whilst the molecules used are well known, in this particular situation of COVID-19, these molecules are prescribed "off-label" in an unconventional treatment and administered to patients infected with a new SARS-CoV-2 whose physiopathology, evolution and complications are not yet well elucidated and characterized by inter-individual, genetic and ethnic variabilities. All this might influence the

\footnotetext{
* Address correspondence to this author at Faculty of Medicine and Pharmacy of Casablanca, Laboratory of Pharmacology-Toxicology, Hassan II University of Casabalnca, Casablanca 2000, Morocco; E-mail: younesskadil@gmail.com
}

discussion of the risk/benefit balance of treatments proposed in those conditions, whereby other factors are involved, paraded by the public health interest and the medical service in terms of therapeutic usefulness.

To identify and approve all adverse events declarations under the COVID-19, the pharmacovigilance expert should collaborate with the units involved in the hospitalization of the patients in question.

As a rule, pharmacovigilance activity is based on the spontaneous notification of undesirable events related to the use of medicinal substances. Considering the special circumstances of the pandemic, many health authorities worldwide have advocated surveillance and monitoring based principally on two types of notifications:

- The encouraged notification consists of inquiring each person with the COVID-19 positive patients if they have observed a wanted ADR.

- The enhanced notification, in which the requirement for information on drug tolerance is referred to the physicians.

The notification document has to include the following essential elements:

- Patient information: The first three letters of the patient's name (to guarantee anonymity), date of birth, sex, weight, and any history of pathological conditions that may be a contributing factor to the adverse reaction, to be included. Thus, this information will allow targeting the occurrence of the most common Adverse Side Reactions (ADR) in specified populations.

- Drug information: The different drugs that were used before the ADR and their dosage, the date on which they were first taken, the date on which the reaction first occurred, and the date on which the treatment was suspended or completed. 
- The description of the suspected adverse event, the time of occurrence after starting the drug, the discontinuation of the drug (if applicable), restorative treatment (if applicable), and the notion of readministration if it has been applied.

- The plausible differential diagnoses, including data from examinations that have been performed.

- The associated factors that may increase the risk or may predispose to the onset of the adverse reaction.

- The evolution of the adverse reaction reported by the medical staff.

The estimation of the scores related to the severity of the effect, as described in Good Pharmacovigilance Practice (GPP), as well as in the legislation texts, cannot be fully strictly applicable in the pandemic situation. This is due to the specificities of the COVID-19 patients who are being managed, particularly in resuscitation and intensive care settings. Most of these patients are polypathological with high-risk conditions (Diabetes, hypertension, etc.) and/or with a severe COVID-19 condition. Moreover, the criteria and the different aspects of the pathology itself remain partially known to date. Furthermore, the contribution of drugs to the occurrence of certain clinical and/or biological effects seem difficult to extricate, linked to the pathology, given the panoply of drugs used in combination to treat patients with a serious prognosis. Thus, we have suggested that an ADR is deemed serious if it is an adverse reaction that is directly life-threatening, or result in significant long-lasting disability or incapacity. For others, the criteria of the judgement of severity, which are prolonged hospitalization, we have discussed its non-adaptation to the present circumstances, considering that the average length of hospitalization of a COVID-19 patient has not yet been estimated and validated. For the last criteria related to the occurrence of a congenital anomaly or malformation, a multidisciplinary staff should discuss the balance of benefits of treatment for the mother and the fetus.

The pharmacovigilance unit will then analyze the report and investigate the causal correlation between each symptom and each drug administered to the patient, using at least two standardized and harmonized methods of imputability [1].

Drugs are classified according to the ATC (Anatomical, Therapeutic and Chemical) classification, which is the most recognized. This classification consists of 5 levels:

- $\quad 1^{\text {st }}$ level: It defines the anatomical group.

- $2^{\text {nd }}$ level: It defines the therapeutic or pharmacological sub-group.

- $3^{\text {rd }}$ level and $4^{\text {th }}$ level: They define the pharmacological, chemical, or therapeutic sub-group.

- $\quad 5^{\text {th }}$ level: It specifies the chemical substance.

For harmonization of notifications, ADRs are coded in the database according to the MedRA classification (Medical Dictionary for Regulatory Activities) [2]. This classification is organized into five levels ranging from the broadest to the most specific. The first level is entitled "System Organ Classes" (SOC), the second "High-Level Group Terms" (HLGT), the third "High-Level Terms" (HLT), the fourth "Preferred Term" (PT) and the last "Lowest Level Terms" (LLT).

It allows an agreement of the standard terminologies used in VigiFlow [3]. VigiFlow is a web-based system for managing and reporting ADR cases internationally. In principle, notified and validated cases will be transmitted to the national pharmacovigilance center via Vigiflow, which in turn forwards reports to the international pharmacovigilance center so that they can be centralized in the international VIGIBASE database. VIGIBASE is a World Health Organization pharmacovigilance database that has been in existence since 1968 and lists more than 8 million reports of Individual Case Safety Reports from various countries. Research and analysis of VIGIBASE are carried out using a search tool: Vigilyze ${ }^{\mathrm{TM}}$ [4]. This tool is used to describe and investigate adverse drug reactions on a global level. The analysis of adverse reactions reported with the drugs that were used for COVID-19 must take into account the context of risk association due to polymedication, associated co-morbidities and without exclusion of the COVID-19 disease itself, whose semiological and evolutionary criteria are not well understood.

It should be noted that any causal relationship between drug intake and adverse reaction is established at a given time and may evolve depending on additional information for the effect that has appeared and/or the drug consumed.

\section{CONSENT FOR PUBLICATION}

Not applicable.

\section{FUNDING}

None.

\section{CONFLICT OF INTEREST}

The authors declare no conflict of interest, financial or otherwise.

\section{ACKNOWLEDGEMENTS}

Declared none.

\section{REFERENCES}

[1] World Health Organization causality assessment method http://www.who-umc.org/ defs.html2002.

[2] Brown EG, Wood L, Wood S. The medical dictionary for regulatory activities (MedDRA). Drug Saf 1999; 20(2): 109-17. [http://dx.doi.org/10.2165/00002018-199920020-00002] [PMID: 10082069]

[3] VigiFlow ${ }^{\mathrm{TM}}$

http://www.whoumc.org/DynPage.aspx?id=97223\&mn1=7347\&

[4] https://www.who-umc.org/vigibase/vigilyze/ 